\title{
Diminished fracture initiation sites in ceramic layers bonded to glow-discharge treated substructure
}

\author{
Hyeongil Kim ${ }^{1}{ }^{*}$, Edward A. Monaco Jr. ${ }^{1}$, Frederick McIntyre ${ }^{2}$, Elaine L. Davis ${ }^{3}$, Robert E. Baier ${ }^{3}$ \\ ${ }^{1}$ Department of Restorative Dentistry, University at Buffalo, New York, USA \\ ${ }^{2}$ VA Western New York Healthcare System at Buffalo, New York, USA \\ ${ }^{3}$ Department of Oral Diagnostic Sciences, University at Buffalo, New York, USA \\ Email: ${ }^{*}$ hik@buffalo.edu \\ Received 10 June 2013; revised 10 July 2013; accepted 31 July 2013 \\ Copyright (C) 2013 Hyeongil Kim et al. This is an open access article distributed under the Creative Commons Attribution License, \\ which permits unrestricted use, distribution, and reproduction in any medium, provided the original work is properly cited.
}

\begin{abstract}
A mechanically retentive structure and meticulous surface cleanliness are critical factors in providing fracture resistance and clinical success of metal ceramic restorations. This investigation compared the porcelain/metal interfaces of deliberate compressive fractures of ceramic crowns between conventional preparation and application of the Radio Frequency Glow Discharge Treatment (RFGDT) before each bonding step. It evaluated RFGDT's capacity to improve wetting effectiveness and minimize porosity. Twelve metal ceramic crowns were fabricated identically. RFGDT was applied to the metal substructures of half the specimens before the ceramic layering process. All specimens were fractured in the same manner by an applied compressive force to simulate dental occlusive failure. Fracture surfaces were inspected by light and scanning electron microscopy. Quantitative analyses of images were performed to identify numbers, locations of cracks, porosity patterns, and other morphological correlates of the fracture zones. There were significantly fewer voids per millimeter at the interfaces in the RFGDT group than in the non-RFGDT group $(t=2.377, d f=9, p=$ 0.021 ). There was a significant difference in the number of horizontal cracks per millimeter between the groups $(t=2.132, d f=7, p=0.035)$, with more cracks occurring in the non-RFGDT group. RFGDT can improve the integrity of metal ceramic crowns by increasing the substratum surface energy, improving porcelain wetting and spreading and thereby diminishing the numbers of interfacial voids available for initiation of fracture. Routine application of RFGDT should result in fewer cracks along metal/ceramic interfaces in all restorative preparations.
\end{abstract}

${ }^{*}$ Corresponding author.
Keywords: Porcelain Fracture; Glow Discharge Treatment; Metal Ceramic Crown; Porosity; Surface Energy

\section{INTRODUCTION}

Ceramics are well known for their natural appearance, durable chemical and optical properties, and as dental restorative materials are frequent replacements for natural teeth because of their good wear resistance, chemical inertness, surface texture and esthetics. A significant early development in the use of porcelain for dental restorations was the fusion of porcelain to gold alloys to overcome inherent brittle failure of ceramic crowns $[1,2]$. The fusion of porcelain to gold alloys allowed the esthetics of porcelain to be combined with the ductility, strength, and toughness of the gold alloy. Restorations fabricated with gold-dominated alloys and porcelain, although clinically reliable, are being replaced with less expensive metal alloy systems that also claim superior physical properties in terms of strength and sag resistance $[3,4]$. In recent years, dental porcelain and alloys for bonding have undergone reformulation, resulting in a greater variety of dental porcelains and ceramic casting alloys, as well as a wider range of compositions and costs. A remaining concern within the art and science of casting, metal work, and porcelain bonding mechanisms is the susceptibility of these systems to brittle fracture at the ceramic/metal interface. In addition to structural soundness, biologic and esthetic expectations of the patient require the resolution of issues concerning the discoloration of silver-containing metal alloys, metal distortion after coldwork [5], marginal discrepancies after porcelain veneering [6-8], and the biological safety of nickel [9] and beryllium-containing base metal alloys [10].

Alternative restorative systems have been developed to overcome limitations of the conventional metal ce- 
ramic restoration. Some systems based on non-metal or all ceramic materials have been developed with excellent esthetic results [11,12]. Other systems have utilized metal substructures with better esthetics and strength due to high noble components of the chosen alloys $[13,14]$.

This investigation selected one of these newer approaches to the fabrication of metal ceramic restorations, based on a non-cast metal substructure (Captek, Precious Chemicals USA Inc., Florida). The fabrication technique consisted of sintering $\mathrm{Au} / \mathrm{Pt} / \mathrm{Pd}$-containing wax lamina onto a refractory die to fabricate a porous structural network followed by infusion of molten gold. The result was a highly dense, well-fitting metal substructure for porcelain veneering that, because of the absence of a chemically compatible oxide layer on the porcelain-bonding substructure, required a mechanically retentive design and meticulous surface cleanliness as critical factors in providing fracture resistance and clinical success of the final units [15]. A ceramo-metal bonder (Capbond, Precious Chemicals USA Inc., Florida) was applied and fired over the oxide-free gold copings to form an additional 25 -to-35 $\mu \mathrm{m}$ thin layer. The ceramo-metal bonder was designed to provide a structure of fine, gold-rich filaments simulating the optical effect of vital pulp in its light-scattering qualities. The irregular Capbond interior structure was expected to also play a role in sustaining strong mechanical bonds between the porcelain and metal substructure [16].

Radio Frequency Glow Discharge Treatment (RFGDT) process as described by Baier and DePalma (1970) is a method for scrupulously cleaning, surface-activating, and in some cases sterilizing ceramic, metal, and polymer substrata [17,18]. RFGDT technique has been well known for surface cleaning and surface modifications, widely used in fields of biomedical materials [19,20], removing superficial contaminants so that contact angles are decreased and surface energy values are increased, minimizing potential wetting and spreading problems.

The objectives of this study were to characterize the porcelain/metal interface after compressive fracture of these ceramic/metal crowns, and to evaluate whether RFGDT could be used as a method to promote wetting/ spreading effectiveness and to minimize void inclusions and fracture cracks at the porcelain-metal interfaces.

\section{MATERIALS AND METHODS}

\subsection{Specimen Fabrication}

The crown materials used in this study (Captek) were applied following manufacturer's instructions, utilizing an adhesive layer, two metal-impregnated wax lamina (one heat-fused over and into the other), and a top-bonding agent for the final porcelain application.

Twelve identical crowns were first fabricated from a master metal tooth. For six specimens, before the placement of each applied layer, the specimen received RFGDT using the RFGDT unit (Harrick Scientific Corp., Ossining, NY). The other 6 specimens were prepared without RFGDT. Each specimen was treated in an atmosphere of residual air for 2 minutes after the vacuum pressure had reached a level below 0.1 torr. Medium frequency discharge $(35 \mathrm{MHz})$ was applied. Immediately after removal from the vacuum chamber, the specimens were followed by next procedures to avoid possible recontamination from room air. The fabrication of crown specimens was standardized to control processing variables. Opaque porcelain and dentin porcelain (Omega 900, Vident, Brea, Calif) were applied using a jig with a consistent powder/liquid ratio. Final porcelain thickness was measured and adjusted so that all specimens had the same dimensions $(2.0 \mathrm{~mm}$ thickness incisally, $1.5 \mathrm{~mm}$ labially, and $1.2 \mathrm{~mm}$ lingually). Surfaces of all specimens were glazed to remove possible exterior surface flaws.

\subsection{Specimen Test}

An excessive compressive force was applied to the incisal edge of each crown using an analogue force-gage and tool with a tooth-cusp-shaped head to induce mechanical fracture, as illustrated in Figure 1A. The number, size, and distribution of interfacial flaws (voids, cracks) were analyzed from optical microscopic and SEM inspections of the specimens made with or without RFGDT treatment (Figure 1B).

The fractured specimens were embedded in epoxy resin (Epofix, Struers Inc., Cleveland, Ohio) and the

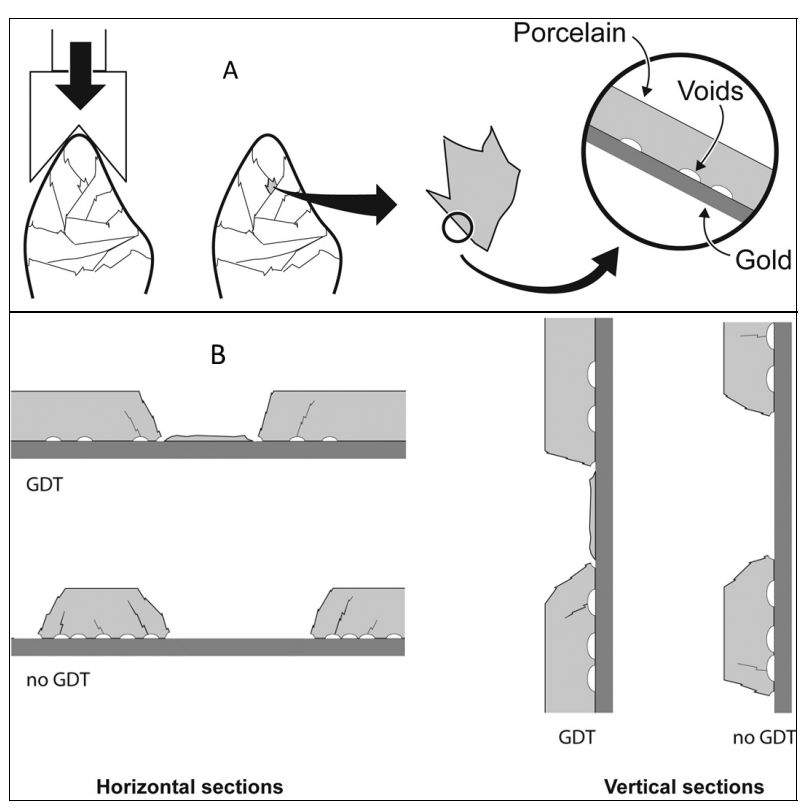

Figure 1. (A) Representation of porcelain mechanical failure from compressive force; (B) Porcelain fracture mode related to interfacial flaws. 
resin-mounted specimens were sectioned in coronal planes through mid-crown areas of porcelain failures with a cross-sectioning diamond disk (Isomet, Buehler, Lake Bluff, Ill). Specimens were analyzed with a field emission SEM (S-4000, Hitachi High Technologies America Inc., Dallas, Tex) at $20 \mathrm{kV}$ operating in secondary electron imaging mode. SEM images were recorded at ten evenly spaced intervals around the perimeter of each cross-section specimen of each of the twelve specimens (Figure 2). Inspections were made of images (300× magnification) of each site for voids, cracks, and other morphological correlates of the fracture zones.

\subsection{Statistical Analysis}

For statistical analysis, all data (number, location, and failure pattern of voids and cracks) were converted to numbers per millimeter of the interfaces examined. Data for the two groups were compared by independent $t$-tests, using a significance level of 0.05 , to determine differences between groups.

\section{RESULTS}

It was readily observed during the fabrication of the specimens that RFGDT of the crowns at each step of preparation resulted in better wetting properties and a more even, thinner layer of the bonding agent when applied to the metal substructure (Figure 3A). NonRFGDT specimens revealed poor distribution of the bonding agent (Figure 3B). All specimens were taken to total mechanical failure, except one of the RFGDT-prepared specimens that resisted the maximum force (approximately 500 Newtons) applied manually during the fracturing process. Mechanical failures included com-

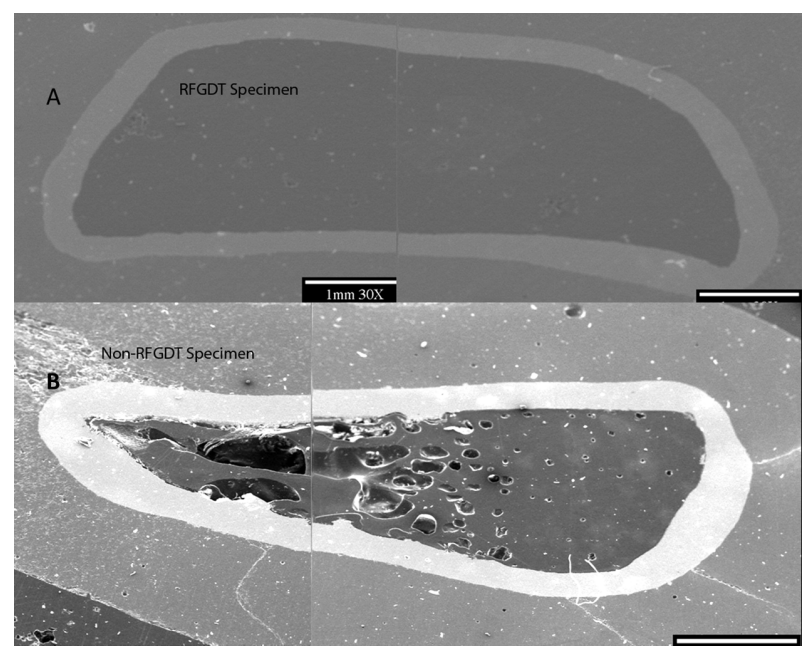

Figure 2. (A) Representative Composite SEM image of cross sectioned ceramic/metal crown prepared with RFGDT; (B) Representative Composite SEM image of cross sectioned ceramic/metal crown prepared with non-RFGDT.

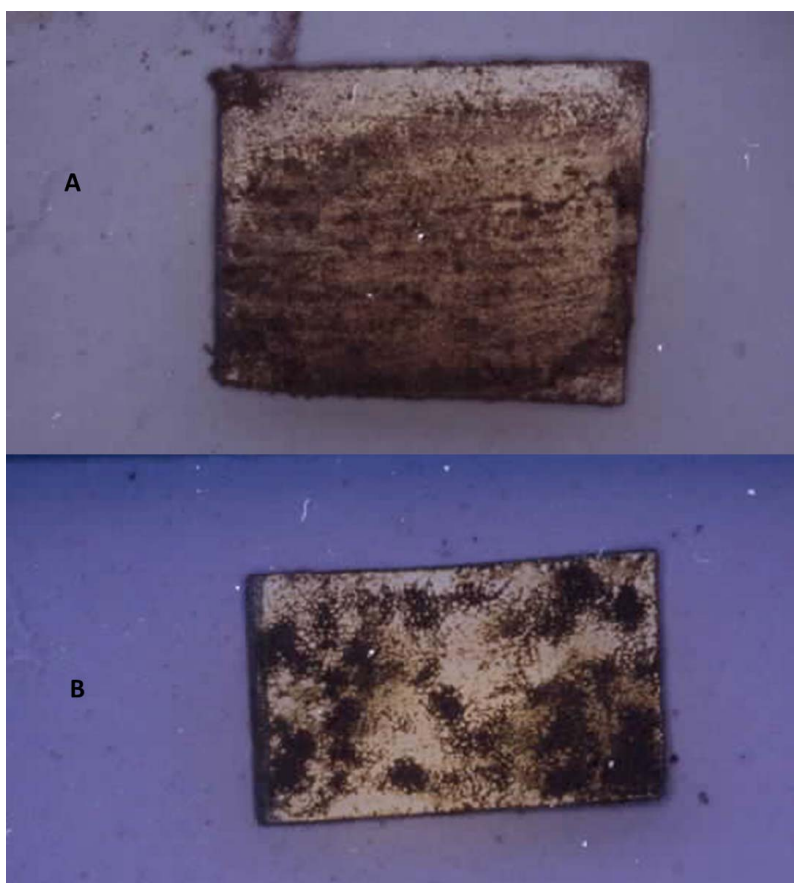

Figure 3. (A) Bonding agent application to RFGDT specimen; (B) Bonding agent application to non-RFGDT specimen.

plete or incomplete cracks, adhesive and cohesive fractures in both RFGDT and non-RFGDT groups.

Visual inspections with the light microscope revealed gross differences between the two specimen groups. The RFGDT group showed mainly small fragments, with failure patterns dominated by microfractures (Figure 4). The non-RFGDT group of specimens showed larger fracture zones revealing more exposed metal under the original porcelain-metal interfaces.

Voids counted during the inspection of each SEM image (total of 120 specimen locations) are enumerated in Tables 1-3. In the non-RFGDT group, from sixty locations on the SEM images that were inspected, a total of 88 voids was recorded. Many voids (44\% of 88 voids) were found at the opaque/bonding agent interface zones. In the RFGDT group, only 48 voids were noted and these were evenly distributed among the metal/opaque/body porcelain interfaces of the sixty locations.

Void sizes measured from each SEM image ranged from 4 to 28 micrometers in the RFGDT group, and 4 to 40 micrometers in the non-RFGDT group.

The total number of cracks observed during SEM image inspections (total of 120 locations) is reported in Tables 4-6. Among the non-RFGDT group, 50 cracks were noted, mostly extending to the bulk from long runs at the metal/ceramic interface. In the RFGDT group, only 35 cracks were found with smaller fractured fragments than in the non-RFGDT group, shorter crack runs along the metal/ceramic/interface were found in the RFGDT group. Since the RFGDT specimen group pre- 
Table 1. Number of voids at porcelain/metal interface.

\begin{tabular}{ccccc}
\hline \multirow{2}{*}{ Group } & \multirow{2}{*}{ Voids } & \multirow{2}{*}{$\begin{array}{c}\text { Mean } \\
\text { voids/specimen }\end{array}$} & \multicolumn{2}{c}{ Voids $/ \mathrm{mm}$ of interface } \\
\cline { 4 - 5 } & & & Mean & SD \\
\hline RFGDT & 22 & 3.67 & 0.150 & 0.920 \\
non-RFGDT & 39 & 6.50 & 0.268 & 0.224 \\
\hline
\end{tabular}

Table 2. Numbers of voids within adjacent opaque layers.

\begin{tabular}{ccccc}
\hline \multirow{2}{*}{ Group } & \multirow{2}{*}{ Voids } & \multirow{2}{*}{$\begin{array}{c}\text { Mean } \\
\text { voids/specimen }\end{array}$} & \multicolumn{2}{c}{ Voids/mm of interface } \\
\cline { 4 - 5 } & & & Mean & SD \\
\hline RFGDT & 26 & 4.33 & 0.178 & 0.150 \\
non-RFGDT & 49 & 8.17 & 0.336 & 0.164 \\
\hline
\end{tabular}

Table 3. Total numbers of boundary layer voids, RFGDT vs. non-RFGDT specimens.

\begin{tabular}{ccccc}
\hline \multirow{2}{*}{ Group } & \multirow{2}{*}{$\begin{array}{c}\text { Total } \\
\text { voids }\end{array}$} & $\begin{array}{c}\text { Mean } \\
\text { voids/specimen }\end{array}$ & \multicolumn{2}{l}{ Voids/mm of interface } \\
\cline { 4 - 5 } & & & Mean $^{*}$ & SD \\
\hline RFGDT & 48 & 8.00 & 0.328 & 0.158 \\
non-RFGDT & 88 & 14.66 & 0.605 & 0.238 \\
\hline
\end{tabular}

*statistically significant $(p<0.05)$.

Table 4. Horizontal interfacial cracks, RFGDT vs. nonRFGDT specimens.

\begin{tabular}{ccccc}
\hline \multirow{2}{*}{ Group } & \multirow{2}{*}{$\begin{array}{c}\text { Hor. } \\
\text { cracks }\end{array}$} & $\begin{array}{c}\text { Mean } \\
\text { Voids/specimen }\end{array}$ & \multicolumn{2}{c}{ Hor. cracks/mm of interface } \\
\cline { 5 - 5 } RFGDT & 3 & 0.50 & Mean $^{*}$ & SD \\
\hline non-RFGDT & 13 & 2.17 & 0.021 & 0.034 \\
\hline
\end{tabular}

"statistically significant $(p<0.05)$.

Table 5. Vertical (bulk material) cracks, RFGDT vs. nonRFGDT specimens.

\begin{tabular}{ccccc}
\hline \multirow{2}{*}{ Group } & \multirow{2}{*}{$\begin{array}{c}\text { Vertical } \\
\text { cracks }\end{array}$} & $\begin{array}{c}\text { Mean } \\
\text { cracks/specimen }\end{array}$ & \multicolumn{2}{l}{ Vertical crack/mm } \\
\cline { 4 - 5 } RFGDT & 32 & 5.33 & 0.219 & 0.109 \\
non-RFGDT & 37 & 6.17 & 0.254 & 0.168 \\
\hline
\end{tabular}

Table 6. Total numbers of cracks, RFGDT vs. non-RFGDT specimens.

\begin{tabular}{ccccc}
\hline \multirow{2}{*}{ Group } & \multirow{2}{*}{$\begin{array}{c}\text { Total } \\
\text { cracks }\end{array}$} & \multirow{2}{*}{$\begin{array}{c}\text { Mean } \\
\text { cracks/specimen }\end{array}$} & \multicolumn{2}{c}{ Crack/mm } \\
\cline { 4 - 5 } RFGDT & 35 & 5.83 & Mean & SD \\
\hline non-RFGDT & 50 & 8.33 & 0.239 & 0.120 \\
\hline
\end{tabular}

sented very little horizontal cracking ( $8.6 \%$ of 35 cracks) along the porcelain/metal interface, microfractures seen away from the interface suggested a predominantly cohesive porcelain fracture pattern. This finding was in

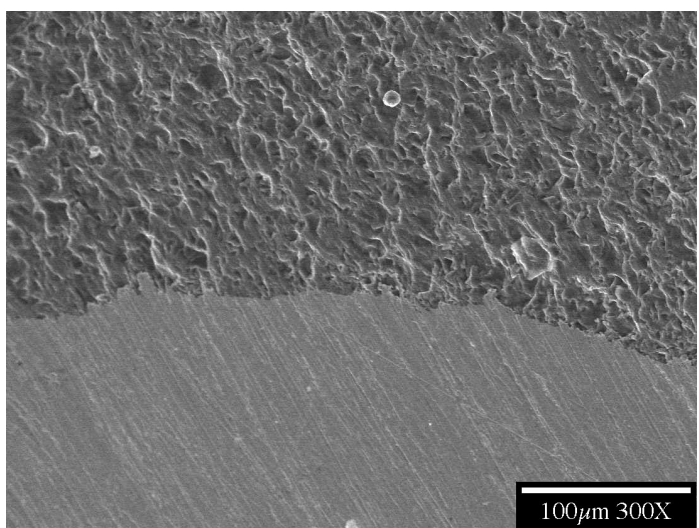

Figure 4. Microfractures with radiant fracture patterns in non-RFGDT group specimen.

strong contrast to the mainly adhesive failures in the nonRFGDT group of specimens. In the non-RFGDT group specimens that revealed more horizontal cracks $(26 \%$ of 50 cracks) than the RFGDT group, most of this horizontal cracking occurred along the metal-porcelain interface.

To affirm that these finding represented equal lengths of specimen interfaces, actual inspected boundaries were measured from all specimen SEM images at 300× magnification. The RFGDT group presented a total length of $24.39 \mathrm{~mm}$ of the interfaces examined. The non-RFGDT group showed a total of $24.27 \mathrm{~mm}$ of the interfaces examined.

When all data were converted to numbers of flaws per millimeter of the interfaces examined, it was clear that there were significantly fewer total voids per millimeter at the RFGDT group interfaces (including the porcelain/ metal interface and the opaque layer) than at the nonRFGDT group $(t=2.377, d f=9, p=0.021)$. There was, similarly, a significant difference in numbers of horizontal cracks per millimeter between the groups $(t=2.132$, $d f=7, p=0.035$ ) with more cracks occurring in the non-RFGDT group. No significant group differences were found for vertical cracks or total numbers of cracks per millimeter $(p>0.05)$, also inspected and counted in each specimen group.

\section{DISCUSSION}

The aim of this work was to diminish the distribution of interfacial flaws in metal ceramic crowns when the RFGDT technique was employed, as illustrated by deliberate fracture under an excessive compressive load applied to the incisal edge (Figure 1A).

Clinicians can face failures of metal ceramic restorations during any step in the fabrication and placement procedure such as tooth reduction, impression making, selection and manipulation of dental materials, laboratory finishing, and cementation of restorations. It is speculated that the diminished presence of internal flaws 
will preserve the structural strengths of restorations, however, the current study's intent was not to measure the failure load. Rather, it enumerates two major factors that enhance the likely clinical success of metal ceramic restorations. The first factor is the intrinsic material properties, differentiated into several categories as Bertolotti described [21]. The chemo-mechanical compatibility of porcelains and casting alloys plays a key role in the success of metal ceramic crowns. It is generally accepted that the three bonding mechanisms of porcelain/ casting alloys are mechanical interlocking, true covalent chemical bonding, and a physical variant of true chemical bonding termed Van der Waals bonding.

Thermal expansion/contraction incompatibility is another important factor in metal ceramic restoration failures. Many studies have shown that failures occur both during fabrication and in the mouth as a result of thermal expansion incompatibility. However, thermal expansion/ contraction values alone are not sufficient to predict thermal expansion compatibility. Thermal history, geometry of prosthesis, and many processing variables are equally important. Other intrinsic factors affecting the strength of metal ceramic restorations are diffusion of metal oxides into porcelain, surface roughness, and interface reactions such that thermodynamic driving forces result in porcelain wetting of the metal and spreading of the molten glass. In previous work, beneficial smaller contact angles have been obtained only when the metal surface was roughened, not recognizing that the lowerthan-ideal degree of surface cleanliness and surface energy was not actually overcome by this process. Here, RFGDT is proposed to be able to enhance the integrity of even very smooth bonding surfaces.

The second factor is extrinsic surface flaws produced by machining, grinding, and other surface treatment methods. The sizes and numbers of extrinsic surface flaws, microcracks, porosities and many different intraoral variables do affect the strength of metal ceramic restorations [22].

Dental porcelains are brittle materials, therefore, fractures occur in porcelain when the applied forces produce stresses at flaw tips equal to the intrinsic tensile strength of porcelain, as described by Griffith [23]. First, the fracture occurs at the most severe flaw present in the stressed region. In dental porcelain, surface flaws are the most important cause of fracture failures [24]. The flaws grow to critical size when placed under increasing stress. A chemical reaction between porcelain and water is responsible for delayed failure in porcelains [25]. The slow extension of the crack continues until the stress intensity at the crack tip reaches a critical value for the particular material. The stress intensity at the crack tip at the time the crack becomes unstable is a material property, termed the "critical stress intensity factor" or "fracture tough- ness". Ceramic strength is directly related to fracture toughness and inversely related to the square root of the flaw size [26]. Flaws can be introduced into a metal ceramic restoration during porcelain powder mixing, buildup, firing, and later contouring, or they can be inherent in the microstructures from grain size and thermal-coefficient mismatches [21]. Kelly et al. analyzed failures of all-ceramic fixed partial dentures (FPDs) in clinical and laboratory situations [26]. They reported that failures originated from either the external surface of connectors of FPDs or from the core-veneer interfaces. For approximately $75 \%$ of all specimens, crack initiation occurred at the core-veneer interface, indicating that the interface is both a location of high tensile stress and an important locus of structural flaws. Interfaces can be the sites of unique defects, boundary phases, and thermal incompatibility stresses due to the elastic modulus mismatches across the interfaces. Scanning electron microscopic observations revealed numerous porous defects in both core and veneer ceramics at the interface. It can be appreciated that restoration failure was originated from the ceramo-metal interface. Kelly et al. also emphasized the importance of flaw size, indicating that calculated tensile strengths of ceramics were reduced with increases of the flaw sizes [26]. Therefore, dental porcelain is very sensitive to flaws in size, number and distribution in the area of highest tensile stress, and the flaws control the material's fracture toughness. In the current work, the observed size of internal voids near the interface between the bonding agent and opaque/dentin porcelain ranged from 4 to 40 micrometers in the non-RFGDT group of specimens, but only from 4 to 28 micrometers in the RFGDT group. Because ceramic materials fail as a result of crack propagation and fracture, this RFGDT-imparted improvement suggests that its increased use during bonding of ceramic materials can lead to improve strength, fracture resistance, and improved performance due to a stronger bond between the porcelain and the metal substructure.

When the bonding agent was applied to the metal substructure in this work, it was observed that RFGDT did promote a more even and thinner layer of that bonding agent. Non-RFGDT specimens showed poorer distribution of the bond layer. Also, the non-RFGDT specimens showed weaker sites of the metal/ceramic restorations, when taken to total mechanical failure, where gross visual and light stereomicroscopic inspection showed substantially larger areas of metal-baring delamination than those of the RFGDT group which presented smaller fractured fragments with crushed failure patterns and fewer areas of metal exposure. Flaws observed at the electron microscope level were defined as follows: Microfractures were radiant fracture patterns near the fractured surfaces or crack lines (Figure 4). Voids were 
smooth-bordered oval-shaped bubbles found on either the metal substructure or in the opaque porcelain layer (Figure 5). Cracks were divided into vertical cracks that extended from the bulk to the metal/porcelain junction and horizontal cracks were those initiated along the metal/porcelain interface (Figure 6). Cross-sectional scanning electron microscope (SEM) images revealed the existence of internal voids between metal substructure/opaque/body porcelain interfaces and/or within porcelain layers. A correlation between the existence of flaw and porcelain failures has been found from the SEM images (Figure 7). The total number of voids per millimeter at the interfaces in the RFGDT group was significantly lower than in the non-RFGDT group. The nonRFGDT group demonstrated more horizontal cracks per millimeter than the RFGDT group, the apparent result of easier porcelain delamination from the metal substructures. Compression-induced vertical fracture cracks were similar in the two test groups, confirming that the bulk material properties had not been modified by the RFGDT process. Thus, it was found that RFGDT decreased interfacial flaws in general, even though there were not

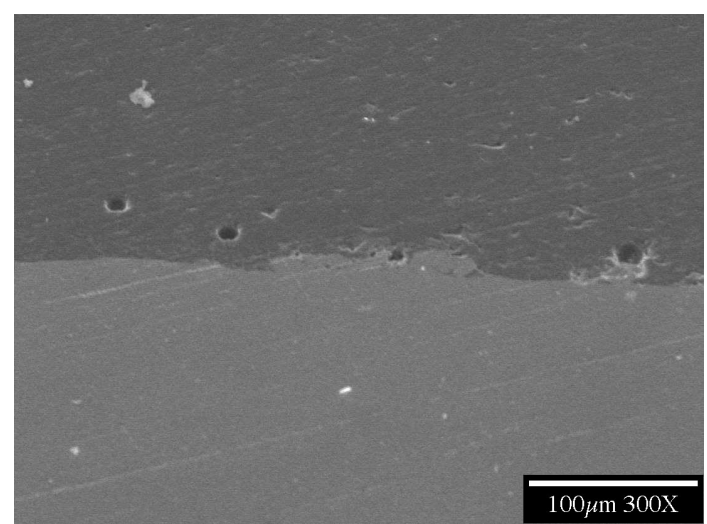

Figure 5. Small voids at the boundary of the dentin and opaque porcelain layers in RFGDT group specimen.

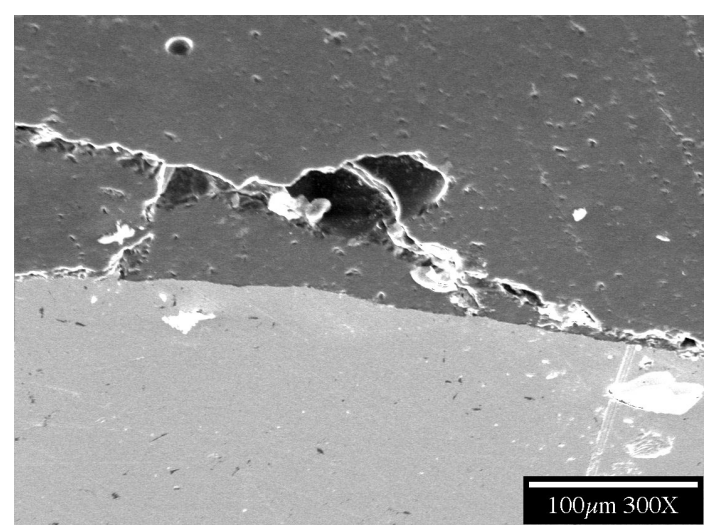

Figure 6. Vertical and horizontal cracks generated along the metal/porcelain interface in non-RFGDT specimen group.

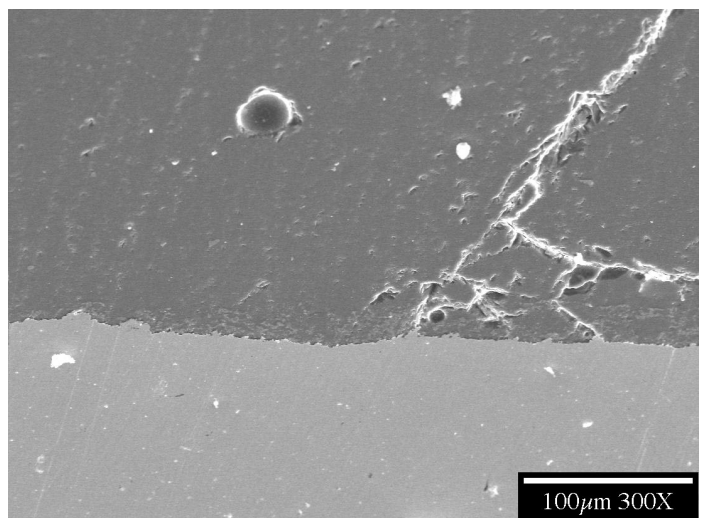

Figure 7. Vertical fracture lines noted in bulk material, propagating to porcelain-metal interface in a nonRFGDT specimen.

statistically significant differences in total numbers of cracks of all types between the two groups. Power analysis, using these already-obtained test results, showed that a larger number of specimens would be required to establish significance in this regard (i.e. A total of 19 specimens are needed to determine differences in total number of cracks between the RFGDT and non-RFGDT groups with $80 \%$ power of achieving statistical significance at $10 \%$ level). Glow-discharge-treatment increased the integrity of the tested crowns mainly by diminishing the number of interfacial voids, and resulted in fewer horizontal cracks along the metal/porcelain interfaces when specimens were broken. Improved integrity of ceramic/metal crown interfaces by glow-discharge-treatment should lead to the better clinical performances, based on these mechanical fracture trials and SEM analyses, and should better maintain the overall integrity of porcelain-metal interfaces during functional loading.

\section{CONCLUSIONS}

Within the limits of this investigation, it is possible to conclude the following:

1) Mechanical fracture of ceramic/metal crowns leads to complete and incomplete cracks as well as adhesive and cohesive fractures, with differences in distribution of the initiating sites attainable by surface energy modification of the bonding faces during the preparation processes.

2) Glow-discharge-treatment at each step of ceramic/ metal fabrication increases the integrity of ceramo-metal interface zones by diminishing the numbers of interfacial voids.

3) Improved integrity of ceramic/metal crown interfaces, achieved by RFGDT, should result in better clinical performances under higher compressive forces.

\section{ACKNOWLEDGEMENTS}

The authors thank Precious Chemicals Inc. for donating Captek ${ }^{\mathrm{TM}}$ 
materials for this study, and Mr. Peter J. Bush in the UB South Campus Instrument Center for his assistance with Scanning Electron Microscopy.

\section{REFERENCES}

[1] Brecker, C.S. (1956) Porcelain baked to gold: A new medium in prosthodontics. Journal of Prosthetic Dentistry, 6, 801. doi:10.1016/0022-3913(56)90077-4

[2] Johnston, J., Dykema, R. and Cunningham, D. (1956) The use and construction of gold crowns with a fused porcelain veneer-A progress report. Journal of Prosthetic Dentistry, 6, 811-821. doi:10.1016/0022-3913(56)90078-6

[3] Anusavice, K.J. (1985) Noble metal alloys for metalceramic restorations. The Dental Clinics of North America, 29, 789-803.

[4] Anusavice, K.J. (1983) Screening tests for metal-ceramic systems. In: McLean, J.W., Ed., Dental Ceramics Proceedings of the First International Symposium on $\mathrm{Ce}$ ramics, Quintessence, Chicago, 371-414.

[5] Campbell, S.D. and Sirakian, A. (1995) Effect of firing cycle and surface finishing on distortion of metal ceramic castings. Journal of Prosthetic Dentistry, 74, 476-481. doi:10.1016/S0022-3913(05)80348-8

[6] Campbell, S.D. and Pelletier, L. (1992) Thermal cycling distortion of metal ceramics: Part I- Metal collar width. Journal of Prosthetic Dentistry, 67, 603-608. doi:10.1016/0022-3913(92)90155-4

[7] Campbell, S.D. and Pelletier, L. (1992) Thermal cycling distortion of metal ceramics: Part II- Etiology. Journal of Prosthetic Dentistry, 68, 284-289. doi:10.1016/0022-3913(92)90331-4

[8] Bridger, D.V. and Nicholls, J.I. (1981) Distortion of ceramometal fixed partial dentures during the firing cycle. Journal of Prosthetic Dentistry, 45, 507-514. doi:10.1016/0022-3913(81)90036-6

[9] Wiltshire, W.A., Ferreira, M.R. and Ligthelm, A.J. (1996) Allergies to Dental Materials. Quintessence International, 27, 513-520.

[10] Pierce, L.H. and Goodkind, R.J. (1989) A status report of possible risks of base metal alloys and their component. Journal of Prosthetic Dentistry, 62, 234-237. doi:10.1016/0022-3913(89)90320-X

[11] Della Bona, A. and Kelly, J.R. (2008) The clinical success of all-ceramic restorations. Journal of the American Dental Association, 139, 85-135.

[12] Wall, J.G. and Cipra, D.L. (1992) Alternative crown systems. Is the metal-ceramic crown always the restoration of choice? The Dental Clinics of North America, 36, 765-782.

[13] Erpenstein, H., Borchard, R. and Kerschbaum, T. (2000)
Long-term clinical results of galvano-ceramic and glassceramic individual crowns. Journal of Prosthetic Dentistry, 83, 530-534. doi:10.1016/S0022-3913(00)70010-2

[14] Shoher, I. and Whiteman, A. (1995 ) Captek-A new capillary casting technology for ceramometal restorations. Quintessence of Dental Technology, 18, 9-20.

[15] Zappala, C., Shoher, I. and Battaini, P. (1996) Microstructural aspects of the Captek alloy for Porcelain- fused-tometal restorations. Journal of Esthetic Dentistry, 8, 151156. doi:10.1111/j.1708-8240.1996.tb00419.x

[16] Shoher, I. (1998) Vital tooth esthetics in Captek restorations. The Dental Clinics of North America, 42, 713-718.

[17] Baier, R.E. and DePalma, V.A. (1970) Electrodeless glow discharge cleaning and activation of high-energy substrates to insure their freedom from organic contamination and their receptivity for adhesives and coatings. Calspan Advanced Technology Center, New York.

[18] Baier, R.E. (1990) Glow-Discharge-Treatment techniques, improvement of adhesion in the intraoral environment by glow-discharge-treatment (GDT) Techniques. Transactions of the Academy of Dental Materials, 3, 6-29.

[19] Tran, H.S., Puc, M.M., Hewitt, C.W., Soll, D.B., Marra, S.W., Simonetti, V.A., Cilley, J.H. and DelRossi, A.J. (1999) Diamond-like carbon coating and plasma or glow discharge treatment of Mechanical heart valves. Journal of Investigative Surgery, 12, 133-140. doi:10.1080/089419399272520

[20] Ramsey, W.S., Hertl, W., Nowlan, E.D. and Binkowski, N.J. (1984) Surface treatments and cell attachment, Tissue Culture Association, Inc. IN VITRO, 20, 802-808. doi:10.1007/BF02618296

[21] Bertolotti, R. (1983) Porcelain-to-metal bonding and compatibility. In: McLean, J.W., Ed., Dental Ceramics Proceedings of the First International Symposium on Ceramics, Quintessence, Chicago, 415-429.

[22] Kelly, J.R. (1995) Perspectives on strength. Dental Materials, 11, 103-110. doi:10.1016/0109-5641(95)80043-3

[23] Kelly, J.R., Giordano, R., Pober, R. and Cima, M.J. (1990) Fracture surface analysis of dental ceramics: Clinically failed restorations. International Journal of Prosthodontics, 3, 430-440.

[24] Southan, D.E. (1983) The Porcelain jacket crown. In: McLean, J.W., Ed., Dental Ceramics Proceedings of the First International Symposium on Ceramics, Quintessence, Chicago, 207-230.

[25] Kelly, J.R. (1999) Clinically relevant approach to failure testing of all-ceramic restorations. Journal of Prosthetic Dentistry, 81, 652-661. doi:10.1016/S0022-3913(99)70103-4

[26] Kelly, J.R., Tesk, J.A. and Sorensen, J.A. (1995) Failure of all-ceramic fixed partial dentures in in vitro and in vivo: Analysis and modeling. Journal of Dental Research, 74, 1253-1258. doi:10.1177/00220345950740060301 